\title{
Simulação da Recuperação de Óleo Através da Injeção de Bancos
}

J.R. NOGUEIRA, Departamento de Matemática, FCT, UNESP, 19060-900 Presidente Prudente, SP, Brasil

M.C. CUNHA, Departamento de Matemática Aplicada, IMECC, UNICAMP, Cx.P. 6065, 13083-970 Campinas, SP, Brasil.

\begin{abstract}
Resumo. Neste trabalho abordamos o problema da recuperação de petróleo por meio da injeção de bancos de água e água com polímero. Estamos interessados em resolver o sistema de leis de conservação dado por equações diferenciais parciais com condições iniciais e de fronteira apropriadas. Usando a solução analítica do problema de Riemann dada em Isaacson (1980) apresentamos um algoritmo que descreve uma solução para o problema da injeção de bancos. As maiores dificuldades na resolução são o tratamento das descontinuidades que surgem devido as interações entre ondas de rarefação e ondas de choque e as dificuldades de implementação numérica da solução.
\end{abstract}

\section{Introdução}

Um processo de recuperação secundária do petróleo consiste na injeção de água no reservatório durante um certo período de tempo, seguida da injeção da mistura de água com polímero. De um modo geral, podemos injetar alternadamente vários bancos de água, com ou sem polímero. A injeção de polímeros melhora a eficiência da recuperação em comparação com o da injeção somente de água, como mostraremos no decorrer do nosso trabalho. A injeção de bancos é economicamente viável, já que a injeção contínua de água com polímeros encarece o processo.

Nosso objetivo é desenvolver e analisar algoritmos que possam ser usados na simulação deste processo. Matematicamente o processo de injeção de bancos (por exemplo 2 bancos), fica caracterizado pelo sistema de equações

$$
\left\{\begin{array}{l}
\frac{\partial s}{\partial t}+\frac{\partial}{\partial x} f(s, c)=0 \\
\frac{\partial}{\partial t}(s . c)+\frac{\partial}{\partial x}(c . f(s, c))=0
\end{array},\right.
$$

com condições iniciais (c.i.) e de contorno (c.c.) do tipo:

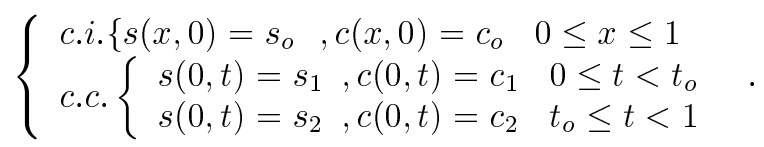


As equações diferenciais que governam o fluxo bifásico água - óleo, são obtidas da Lei de Darcy e da conservação das fases. Neste modelo admitimos que as forças gravitacionais e capilares podem ser desprezadas.

A primeira equação do sistema (1.1) modela a conservação da água no fluxo bifásico e é conhecida como equação de Buckley-Leverett (ver [2]). A Segunda equação modela a conservação do polímero, que por hipótese é solúvel na água. As incógnitas do modelo são a saturação da água, dada por $s(x, t)$ e a concentração de polímero, dada por $c(x, t)$.

As soluções do Problema de Riemann apresentadas por Isaacson [2] são de fundamental importância para o desenvolvimento deste trabalho. Para o caso da injeção de bancos, além das descontinuidades descritas pela solução do problema de Riemann, novas descontinuidades se formam com a interação entre as s-ondas e as c-ondas que aparecem na solução global do problema.

Neste trabalho apresentamos os procedimentos de cálculo utilizados na construção de uma solução semi-analítica, bem como a implementação numérica visando simulações deste processo de recuperação.

\section{Adimensionalização e cálculos numéricos}

Nas aplicações práticas vimos que a saturação de água varia entre uma saturação mínima $s_{w i}$ e uma saturação máxima dada pela diferença $1-s_{o r}$, onde $s_{o r}$ é a saturação de óleo residual. Para facilitar nossos cálculos é conveniente tomarmos uma mudança de variável que faça a saturação $s(x, t)$ variar no intervalo unitário $I=[0,1]$.

A primeira equação do sistema (1.1) modela a conservação da água no fluxo bifásico e é conhecida como equação de Buckley Leverett e é dada por

$$
\frac{\partial s_{w}}{\partial t_{D}}+\frac{\partial f_{1}\left(s_{w}, c\right)}{\partial x_{D}}=0, s_{w i} \leq s_{w} \leq 1-s_{o r}
$$

onde as variáveis independentes foram adimensionalizadas por

$$
x_{D}=\frac{x}{L} \quad e \quad t_{D}=\frac{U . t}{\phi L} .
$$

Para simplificar anotação vamos omitir $D$ na equação (2.1) isto é, daqui para frente $x$ e $t$ representam as variáveis adimensionalizadas.

Podemos verificar facilmente que a mudança de variáveis $s_{w}=s_{n} . s+s_{w i}$ com $s_{n}=1-s_{w i}-s_{\text {or }}$ transforma o intervalo $s_{w i} \leq s_{w} \leq 1-s_{\text {or }}$ no intervalo $0 \leq s \leq 1$ onde a variável $s$ será chamada de saturação normalizada.

Com a mudança de variáveis a equação da conservação da água (2.1) fica reescrita como

$$
s_{n} \frac{\partial s}{\partial t}+\frac{\partial f(s, c)}{\partial x}=0
$$

onde $0 \leq s \leq 1$ e $f(s, c)=f_{1}\left(s_{w}(s), c\right)$. 
A equação da conservação do polímero fica da forma:

$$
\left(s+\frac{s_{w i}}{s_{n}}\right) s_{n} \frac{\partial c}{\partial t}+f(s, c) \frac{\partial c}{\partial x}=0 .
$$

Por conveniência vamos tomar uma nova mudança de variável em $t$, dada por $t=\frac{t_{\text {antigo }}}{s_{n}}$. Assim ficamos com a seguinte equação:

$$
\left(s+\frac{s_{w i}}{s_{n}}\right) \frac{\partial c}{\partial t}+f(s, c) \frac{\partial c}{\partial x}=0 .
$$

O sistema (1.1), adimensionalizado fica escrito então na seguinte forma matricial:

$$
\left[\begin{array}{l}
\frac{\partial s}{\partial t} \\
\frac{\partial c}{\partial t}
\end{array}\right]+\left[\begin{array}{cc}
\frac{\partial f}{\partial s} & \frac{\partial f}{\partial f} \\
0 & \frac{f}{s+s_{w i} / s_{n}}
\end{array}\right]\left[\begin{array}{l}
\frac{\partial s}{\partial x} \\
\frac{\partial c}{\partial x}
\end{array}\right]=\left[\begin{array}{l}
0 \\
0
\end{array}\right] .
$$

Os autovalores deste sistema são

$$
\lambda_{S}(s, c)=\frac{\partial f}{\partial s}(s, c) \text { e } \lambda_{\mathrm{C}}(\mathrm{s}, \mathrm{c})=\frac{\mathrm{f}(\mathrm{s}, \mathrm{c})}{\mathrm{s}+\mathrm{s}_{\mathrm{wi}} / \mathrm{s}_{\mathrm{n}}} .
$$

\section{Algoritmo para a solução}

A dificuldade em se obter uma solução para o caso da injeção de bancos é tratar as descontinuidades que surgem na solução global. No trabalho de Isaacson [2] são tratadas as descontinuidades inerentes a solução do problema de Riemann associados a leis de conservação não convexa, (equação de Buckley-Leverett) devido ao formato em $S$ da função de fluxo fracionário.

A proposta é desenvolver uma sistemática para o cálculo da solução semi analítica que estará fundamentado nas soluções básicas do problema de Riemann dadas por Isaccson. Na realidade a estratégia é trabalhar com problemas de Riemann locais.

Para um melhor entendimento do procedimento consideramos inicialmente o modelo com injeção de dois bancos sendo o primeiro de água seguido de outro de água com polímero.

Vamos supor um reservatório que inicialmente não contenha água e nem polímero, ou seja, $s_{o}=0$ e $c_{o}=0$. Durante um período de tempo $t_{o}$ injetamos água sem polímero $\left(c_{1}=0\right)$ e a partir daí passamos a injetar a mistura de água com concentração de polímero $c_{2}>0$.

Vamos fazer uma análise da solução em duas etapas: a injeção do primeiro banco $\left(t<t_{o}\right)$ e em seguida a injeção do segundo banco $\left(t \geq t_{o}\right)$.

\subsection{A injeção do primeiro banco $\left(t<t_{o}\right)$}

Para $t<t_{o}$ a solução está bem caracterizada, pois trata-se da bem conhecida solução da equação de Buckley-Leverett que consiste da composição de uma s-rarefação e um s-choque.

A Figura 1, mostra a configuração das características no espaço $(x, t)$ quando $t<t_{o}$. 


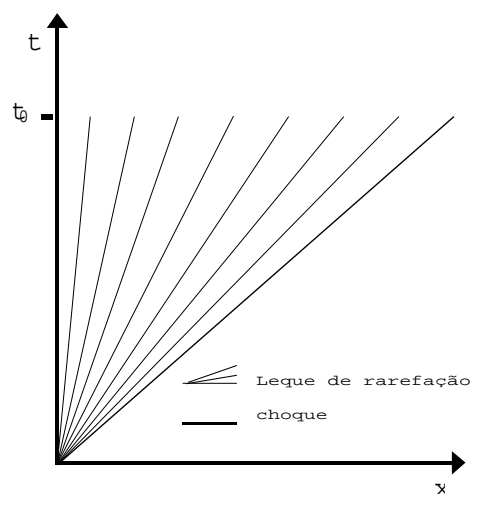

Figura 1

\subsection{A injeção do segundo banco $\left(t \geq t_{0}\right)$}

Quando $t=t_{0}$ paramos de injetar água e começamos a injetar a mistura água com polímero. Temos portanto uma descontinuidade na concentração $c(0, t)$ no ponto de injeção, pois passamos de $c_{1}=0$ para $c_{2}>0$. Esta descontinuidade se propagará no interior do plano $x t$ como mostraremos logo mais. De acordo com Isaacson [2] a solução do problema de Riemann dependerá da localização dos estados à direita $u_{d}=\left(s_{d}, c_{d}\right)$ e estados à esquerda $u_{e}=\left(s_{e}, c_{e}\right)$ no plano das fases.

Consideremos a situação em que o valor da concentração à direita $c_{d}$ seja menor que o da esquerda $c_{e}$ e que o estado $u_{e}$ seja tal que pertence à região $R=\{u=$ $\left.(s, c) \mid \lambda_{S}(s, c)<\lambda_{C}(s, c)\right\}$.

As possíveis seqüências de ondas compatíveis que compõe a solução neste caso são

$$
u_{e} \stackrel{S}{\longrightarrow} u^{1} \stackrel{C}{\longrightarrow} u_{d} \quad \text { se } \quad \mathrm{s}_{\mathrm{d}} \geq \mathrm{s}_{\mathrm{k}}
$$

$$
u_{e} \stackrel{S}{\longrightarrow} u^{*} \stackrel{C}{\longrightarrow} u^{2} \stackrel{S}{\longrightarrow} u_{d} \quad \text { se } \quad \mathrm{s}_{\mathrm{d}}<\mathrm{s}_{\mathrm{k}} .
$$

Como ilustrado na Figura 1 vamos considerar $n$ retas características pertencentes ao leque de rarefação que compõe a solução da equação de Buckley-Leverett. Observamos que para o caso em que um volume poroso é injetado, o valor $n=100$ é satisfatório. De acordo com o método das carcaterísticas cada reta carrega um valor $s$ (saturação) e um valor $c$ (concentração). A inclinação da caractetrística é dada por $\lambda_{S}(s, c)$. A reta que tem inclinação maior, no caso o eixo $t$, carrega o valor $s=1$ e a característica de inclinação menor, carrega o valor $s=s_{m}$. Podemos então associar a estas características um vetor $s_{d}$, com $n$ coordenadas, sendo $s_{d}(1)=1$ e $s_{d}(n)=s_{m}$ e considerar no plano das fases os estados $u_{d}^{j}=\left(s_{d}(j), c_{i n j 1}\right)$, $j=1, \ldots, n$. Para apresentarmos a solução é necessário que calculemos as curvas de descontinuidades decorrentes da injeção do segundo banco. Tais descontinuidades 
surgem a partir da interação entre ondas de rarefação e de choque. Passamos portanto descrever o algoritmo de construção destas curvas, o que será feito em três etapas. Nas duas primeiras etapas descrevemos os procedimentos de cálculo dos dois primeiros pontos, a generalização do procedimento e o cálculo do último ponto. $\mathrm{Na}$ terceira etapa descrevemos as outras curvas de descontinuidades que surgem no problema.

\subsubsection{Etapa 1: Construção de $y_{11}$}

(1.A) Cálculo do primeiro é segundo ponto: Denotemos por $P_{1}$ o ponto inicial da curva de descontinuidade associada a mudança de bancos. Esta curva será denotada pelo par $\left(y_{11}, t_{11}\right)$. Assim o ponto $P_{1}$ é definido pelas coordenadas $\left(y_{11}(1), t_{11}(1)\right)$ que no nosso caso terá valor $\left(0, t_{0}\right)$.

Consideremos o problema de Riemann associado a $\mathrm{P} 1$ com estados à direita e à esquerda dados respectivamente por $u_{d}=u_{d}^{2}=\left(s_{d}(2), 0\right)$ e $u_{e}=\left(s_{2}, c_{2}\right)$

Como vimos a solução para o Problema de Riemann neste caso é do tipo (3.2). $\mathrm{Na}$ notação introduzida acima teremos

$$
u_{i n j 2} \stackrel{S}{\longrightarrow} u_{1}^{2} \stackrel{C}{\longrightarrow} u_{d}^{2} .
$$

O estado intermediário $u_{2}^{1}$ tem coordenadas $\left(s_{1}(2), c_{2}\right)$ onde o valor $s_{1}(2)$ é a solução da equação

$$
\lambda_{C}\left(x, c_{2}\right)=\lambda_{C}\left(u_{d}^{2}\right)
$$

sujeita a restrição

$$
\lambda_{S}\left(x, c_{2}\right) \leq \lambda_{C}\left(x, c_{2}\right)
$$

Consideremos $\mathrm{r}$ a reta com inclinação dada por $\lambda_{C}\left(s_{d}(2), c_{2}\right)$, que passa por $A=(-k, 0)$ e $B=\left(s_{d}(2), f\left(s_{d}(2), 0\right)\right)$,onde $k=\frac{s_{w i}}{s_{n}}$. Geometricamente, $s_{1}(2)$ é a ordenada do ponto de interseção da reta $r$ com o gráfico da função de fluxo fracionário $f\left(s, c_{2}\right)$, como mostra a Figura 2.

$\mathrm{O}$ ponto $P_{2}$ é tomado como sendo a interseção da característica que carrega o estado $u_{d}^{2}$, com o c-choque dado em (3.1) que tem estado a esquerda $u_{1}^{2}$, como mostra a Figura 3 .

Denotamos as coordenadas do ponto $P_{2}$ pelo par $\left(y_{11}(2), t_{11}(2)\right)$.

A solução final $s(x, t)$ e $c(x, t)$ para $t_{0}<t \leq t_{11}(2)$ é representada pela composição

$$
u_{2} \stackrel{S}{\longrightarrow} u_{1}^{2} \stackrel{C}{\longrightarrow} u_{d}^{2} \stackrel{S}{\longrightarrow} u_{o}
$$

(1.B) Cálculo do terceiro ponto: Consideremos o problema de Riemann em $P_{2}$, com estado a esquerda $u_{e}=u_{1}^{2}$ e estado a direita $u_{d}=u_{d}^{3}$. Se ainda tivermos que $s_{d}(3)>s_{k}$ a solução do problema ainda é do tipo (3.2), ou seja, é dada pela composição 


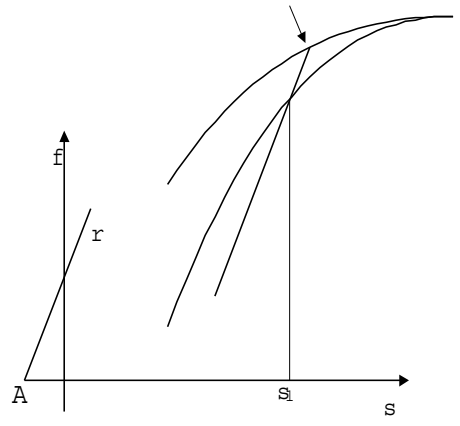

Figura 2

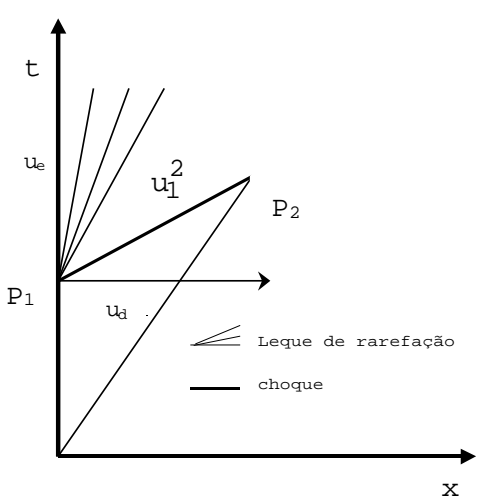

Figura 3

$$
u_{1}^{2} \stackrel{S}{\longrightarrow} u_{1}^{3} \stackrel{C}{\longrightarrow} u_{d}^{3}
$$

onde $u_{1}^{3}$ é o estado intermediário calculado de maneira análoga à $u_{1}^{2}$.

$\mathrm{O}$ ponto $P_{3}$ é tomado como a interseção da reta característica com inclinação com o c-choque que aparece na composição (3.7), como ilustra a Figura 4.

Pode-se verificar pela Figura 4 que a solução $s(x, t)$ e $c(x, t)$ para $t_{11}(2)<t \leq$ $t_{11}(3)$ é dada pela composição

$$
u_{2} \stackrel{S}{\longrightarrow} u_{1}^{3} \stackrel{C}{\longrightarrow} u_{d}^{3} \stackrel{S}{\longrightarrow} u_{o} .
$$

Observação: Lembramos que a primeira s-onda que aparece é uma s-rarefação que liga os estados $u_{i n j 2}$ ao estado $u_{1}^{3}$. A diferença entre (3.6) e (3.8) está no valor da saturação de $u_{1}^{3}$ ser menor que a de $u_{1}^{2}$. De maneira análoga, construímos os demais pontos da curva e paramos quando chegamos $s_{d}(j) \geq s_{k}$. Podemos supor que existe um índice $j_{o}, 1<j_{o}<n$ tal que $s_{d}\left(j_{o}\right)=s_{k}$. De acordo com (3.1) este seria o caso limite, ou seja, o último ponto da curva $y_{11}$. A partir daí a composição da solução passa a ser do tipo (3.2), Sendo assim vamos calcular este ponto separadamente.

(1.C) Cálculo do último ponto: Como $s_{d}\left(j_{o}\right)=s_{k}$, o problema de Riemann é tomado em $P_{j_{o}-1}$ com estado a esquerda $u_{e}=u_{1}^{j_{o}-1}$ e estado a direita $u_{d}=\left(s_{k}, c_{i n j 1}\right)$. A solução ainda é do tipo (3.1) e neste caso

$$
u_{1}^{j_{o}-1} \stackrel{S}{\longrightarrow} u_{1}^{j_{o}} \stackrel{C}{\longrightarrow} u_{d}^{j_{o}},
$$

onde o estado intermedário $u_{1}^{j_{o}}$ é calculado de maneira análoga ao detalhado quando calculamos $u_{1}^{2}$.

Calculamos então o ponto $P_{j_{o}}=\left(y_{11}\left(j_{o}\right), t_{11}\left(j_{o}\right)\right)$ de maneira análoga aos anteriores como mostra a Figura 5. 


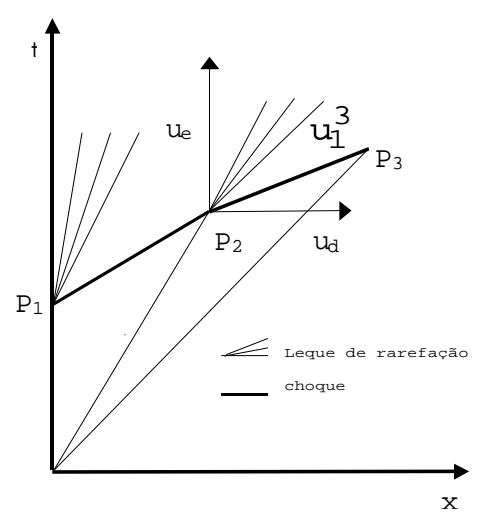

Figura 4

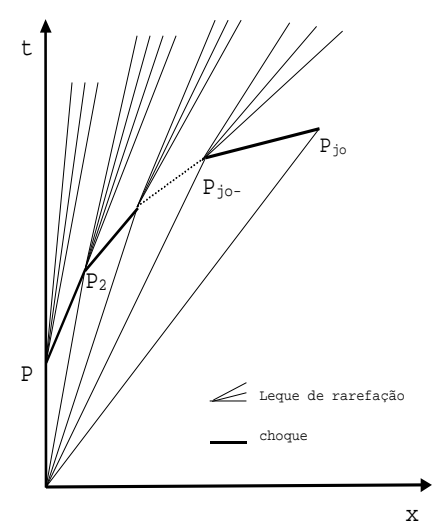

Figura 5

A solução para $t_{11}\left(j_{o}-1\right)<t \leq t_{11}\left(j_{o}\right)$ é dada pela composição

$$
u_{i n j 2} \stackrel{S}{\longrightarrow} u_{1}^{j_{o}} \stackrel{C}{\longrightarrow} u_{d}^{j_{o}} \stackrel{S}{\longrightarrow} u_{o} .
$$

Observamos aqui que a primeira s-onda é uma rarefação começando em $u_{i n j 2}$ e terminando em $u_{1}^{j_{o}}$ (esta rarefação sempre fará parte da composição da solução a partir de $\left.t=t_{11}\left(j_{o}\right)\right)$.

Verificamos que a poligonal que liga os pontos $P_{1}, P_{2}, \ldots, P_{j_{o}}$, aqui denotada por $y_{11}$, é uma curva de choque com estados à direita dados por $u_{d}^{j}, j=1, \ldots, j_{o}$ e estados à esquerda $u_{e}^{1}=u_{1}^{j}$ calculados de maneira análoga à $u_{1}^{2}$ conforme Figura 2.

\subsubsection{Etapa 2: Construção da curva $y_{12}$}

(2.A) Cálculo do primeiro e segundo ponto: Tomemos $Q_{1}$ com coordenadas $\left(y_{12}(1), t_{12}(1)\right)$ como sendo igual ao ultimo ponto da curva $y_{11}$. Portanto $Q_{1}=P_{j_{o}}$. Consideremos então o problema de Riemann em $Q_{1}$ com estado a esquerda $u_{e}=u_{1}^{j_{o}}$ e estado a direita $u_{d}=u_{d}^{j_{o}+1}$. Como $s_{d}\left(j_{o}+1\right)<s_{k}$, a solução agora é do tipo (3.2), ou seja

$$
u_{1}^{j_{o}} \stackrel{S}{\longrightarrow} u^{*} \stackrel{C}{\longrightarrow} u^{2} \stackrel{S}{\longrightarrow} u_{d}^{j_{o}+1} .
$$

Sabemos que em (3.2) s-onda que liga $u_{2}$ a $u_{d}$ é um choque na saturação e que $u_{2}=\left(s_{2}, c_{i n j 1}\right)$, sendo $s_{2}$ tal que, $\lambda_{C}\left(s^{2}, c_{1}\right)=\lambda_{S}\left(s^{*}, c_{2}\right), s^{2}<s^{*}$. Geometricamente, é dado pela interseção da reta que passa por $A=\left(s^{*}, f\left(s^{*}, c_{2}\right)\right.$ e $B=(-k, 0), k=\frac{s_{w i}}{s}$, com o gráfico da função de fluxo fracionário $f\left(s, c_{1}\right)$.

O segundo ponto da curva $y_{12}, Q_{2}=\left(y_{12}(2), t_{12}(2)\right)$ é obtido pela interseção da reta característica com inclinação $\lambda_{S}\left(u_{d}^{j_{0}+1}\right)$ com o s-choque com inclinação dada por $m\left(s_{d}^{j_{0}+1}\right)$ onde $m(x)$ é dada por 


$$
m(x)=\frac{f\left(x, c_{i n j 1}\right)-f\left(s^{2}, c_{i n j 1}\right)}{x-s^{2}} .
$$

A Figura 6 ilustra a situação acima.

A solução global $s(x, t)$ e $c(x, t)$ para $t_{12}(1)<t<t_{12}(2)$ é dada pela composição

$$
u_{i n j 2} \stackrel{S}{\longrightarrow} u^{*} \stackrel{C}{\longrightarrow} u^{2} \stackrel{S}{\longrightarrow} u_{d}^{j_{o}+1} \stackrel{S}{\longrightarrow} u_{o} .
$$

(2.B) Cálculo do terceiro ponto: Consideramos o problema de Riemann sobre $Q_{3}$ com estado à esquerda $u_{e}=u^{2}$ e estado a direita $u_{d}=u_{d}^{j_{o}+2}$. A solução é dada pelo s-choque

$$
u^{2} \stackrel{S}{\longrightarrow} u_{d}^{j_{o}+1}
$$

Calculamos então o valor de $Q_{3}=\left(y_{12}(3), t_{12}(3)\right)$ como sendo a interseção da reta característica com inclinação $\lambda_{S}\left(u_{d}^{j_{0}+2}\right)$ com o s-choque com inclinação $m\left(u_{d}^{j_{0}+1}\right)$. A Figura 7 ilustra esta situação.

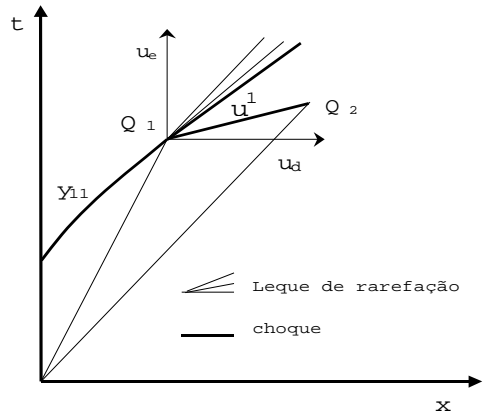

Figura 6

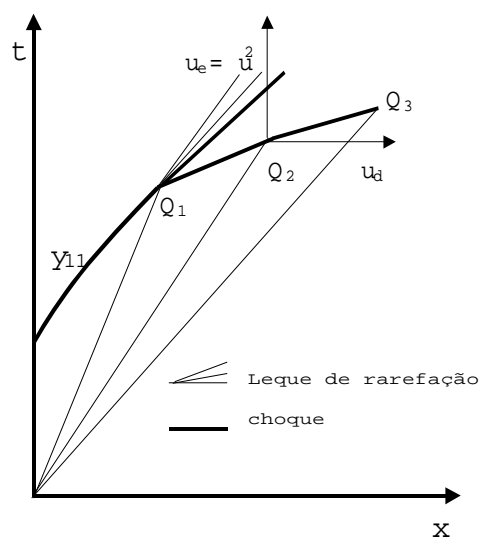

Figura 7

(2.C) Cálculo do último ponto: Continuamos com este procedimento até $j=$ $n$. Neste caso teremos $s_{d}^{n}=s_{m}$. Consideramos o problema de Riemann em $Q_{n-1}=$ $\left(y_{12}(n-1), t_{12}(n-1)\right)$ com estados à esquerda e à direita dados por $u_{e}=\left(s_{2}, c_{i n j 1}\right)$ e $u_{d}=\left(s_{d}^{n}, c_{1}\right)$.

O ponto $Q_{n}$ é obtido pela interseção da reta característica com inclinação $\lambda_{S}\left(u_{d}^{n}\right)$ com o s-choque com inclinação $m\left(u_{d}^{n}\right)$.

Consideremos o problema de Riemann em $Q_{n}$ com estado a esquerda $u_{e}=u^{2}$ e estado a direita $u_{d}=u_{o}$. A solução é dada por um s-choque, neste caso 


$$
u^{2} \stackrel{S}{\longrightarrow} u_{o} .
$$

Este choque é uma reta que se propaga a partir de $Q_{n}$ e será denotada por $y_{13}$ como mostra a Figura 8.

\subsubsection{Etapa 3: Curva $y_{14}$}

A solução está bem definida acima das curvas $y_{11}$ e $y_{12}$ pelas características do leque de rarefação que se formam a partir delas. Dentre elas um outro choque aparece naturalmente na solução final, já que a partir de $t=t_{11}$ (jo) o c-choque

$$
u^{*} \stackrel{C}{\longrightarrow} u^{2} .
$$

sempre faz parte da composição da solução, como vemos em (3.13) Este choque é uma reta que se propaga a partir de $P_{j o}$, com inclinação dada por $\lambda_{S}\left(u^{2}\right)$ e será denotado por $y_{14}$.

Para os dados iniciais propostos podemos mostrar que a velocidade de $y_{13}$ é menor que a velocidade de $y_{14}$ e sendo assim estes dois choques não vão interagir (ver Figura 9).

Com isto descrevemos o algoritmo da construção das curvas de choque para o problema da injeção de bancos. Para completar vamos denotar por $y_{0}$ o choque formado pela equação de Buckley-Leverett.

A Figura 9 mostra a configuração das características e curvas de descontinuidade choque para o problema da injeção de dois bancos, para o caso sem adsorção. Usando esta configuração podemos calcular o valor da saturação e da concentração em cada ponto $(x, t)$ com $t>0$ e $0 \leq x \leq 1$.

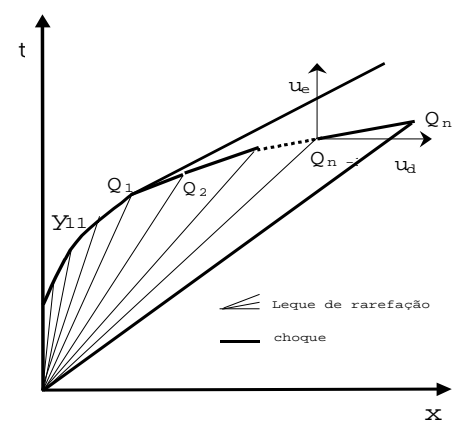

Figura 8

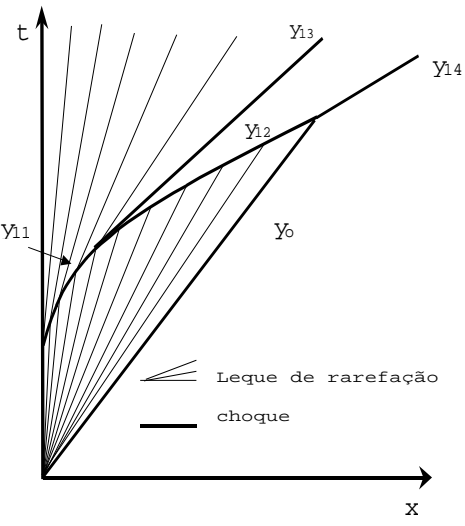

Figura 9 
Observação: Se refinamos o vetor $s_{d}$, ou seja, aumentamos a quantidade de características na rarefação correspondente ao banco de água, as curvas $y_{11}$ e $y_{12}$ tendem á uma configuração mais suavizada.

\section{A injeção de três bancos}

O modelo matemático da injeção de um terceiro banco, consiste em alterarmos a condição de contorno, que agora fica da seguinte forma

$$
\left\{\begin{array}{lll}
s(0, t)=1 & c(0, t)=c_{1} & 0 \leq t<t_{o} \\
s(0, t)=1 & c(0, t)=c_{2} & t_{o} \leq t<t_{1} \\
s(0, t)=1 & c(0, t)=c_{3} & t_{1} \leq t \leq 1
\end{array}\right.
$$

Supondo que a partir de $t=t_{1}$, paramos de injetar água com polímero e voltamos a injetar água sem polímero, devemos ter $c_{1}=0, c_{2}>0$ e $c_{3}=0$. Olhando a configuração final das características para o caso em que dois bancos são injetados (ver Figura 9), observamos que se injetamos um terceiro banco à partir de $t=t_{1}$ uma nova curva de choque se forma pela interação entre as características já existentes na configuração associado a $t<t_{1}$ e as caratcerísticas associadas a injeção do novo banco. Esta nova curva será denotada por $y_{21}$ e sua construção é feita de maneira análoga a curva $y_{11}$, ou seja, considerando problemas de Riemann locais. A Figura 10 mostra uma configuração para as rarefações e choques no caso da injeção de três bancos.

Se injetarmos novos bancos, novas curvas de descontinuidades aparecerão e o tratamento será análogo. Sendo assim a solução semi-analítica para o caso sem adsorção fica bem definida. Esta solução depende exclusivamente do tratamento dado as descontinuidades do problema.

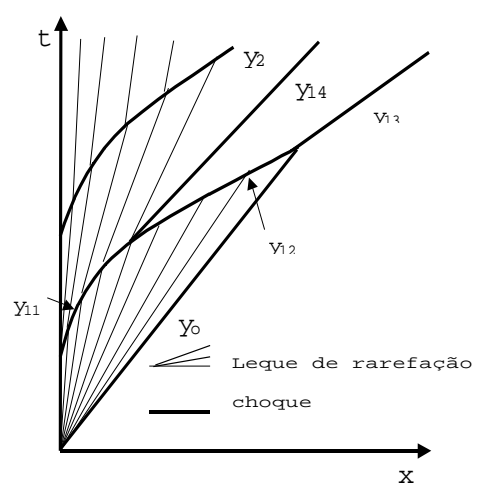

Figura 10 


\section{Testes Numéricos}

Neste parágrafo vamos apresentar alguns resultados obtidos em experimentos numéricos realizados pelo programa computacional desenvolvido. O programa simula a solução para o problema da recuperação de petróleo usando aditivos ativos à água injetada no reservatório.

Utilizamos a seguinte expressão algébrica para simular a função de fluxo fracionário

$$
f(s, c)=\frac{s^{2}}{s^{2}+(k+100 c)(1-s)^{2}},
$$

onde $k$ é uma constante que representa a razão entre as viscosidades, da água e do óleo.

Para calcular a recuperação de óleo nos interessa calcular o valor da saturação de óleo em $x=1$, que de acordo com nossa adimensionalização representa a localização do poço produtor, ou seja, onde mediremos a quantidade de óleo que está sendo produzida pelo processo. somando estas saturações tempo a tempo.

Como nossos programas foram elaborados tendo como incógnita a saturação de água, $s(x, t)$, o cálculo da quantidade de óleo produzida como função do tempo, é medida, de acordo com Marle [6] pela integral

$$
Q_{o}(t)=\int_{0}^{t}(1-f(s(1, x), c(1, x)) d x
$$

que representa o acumulado de óleo recuperado expresso como uma fração do volume poroso.

Esta expressão é usada nos experimentos que apresentamos nesta seção.

\subsection{Experimento 1: Injeção de água com polímeros}

Neste exemplo consideremos um reservatório com as seguintes características: saturação de água contida no reservatório no instante $t=0$ dada por $s_{w i}=0.1$ (significa que $10 \%$ do volume total de fluídos), saturação de óleo residual dada por $s_{\text {or }}=0.1 \mathrm{e}$ concentração de polímero $c=0$.

O programa desenvolvido calcula o valor da saturação $s(x, t)$ baseado na solução analítica do problema de Riemann apresentada por Isaacson [2] e consiste como vimos da composição de s-ondas e c-ondas. Calculamos a produção para valores de $t$ grandes e observamos que quando um volume poroso de fluidos é injetado no reservatório (o que corresponde à $t_{D}=1$ ) a quantidade de óleo resucperado é da ordem de $90 \%$ para o processo de injeção de água com polímero. Para $t_{D}=5 \mathrm{a}$ recuperação é da ordem de $97 \%$. Observamos que para o caso da injeção de água sem polímeros esta recuperação será da ordem de $80 \%$ para $t_{D}=1$. A conclusão é que a recuperação é mais rápida no caso da injeção de água com polímero, ou seja recuperamos uma quantidade de óleo maior num espaço de tempo menor quando comparado com a injeção de água. Isto está ilustrado na Figura 11. 


\subsection{Experimento 2: Comparando com a injeção de bancos}

Vamos considerar a situação em que injetamos $20 \%$ de um volume poroso de água no reservatório e a partir daí injetamos a mistura de água com polímero com uma concentração $c=0.01$. De acordo com os nossos cálculos, injetar $20 \%$ do volume poroso de água corresponde a tomarmos $t_{o}=\frac{0.2}{s_{n}}\left(s_{n}=1-s_{w i}-s_{o r}\right)$ na solução do sistema de equações parciais adimensional.

Utilizando o programa computacional calculamos a evolução da produção de óleo até que 5 volumes porosos de fluido sejam injetados, ou seja até $t_{D}=5$. Os cálculos mostram que a produção converge para a quantidade de óleo máxima $Q_{o}=1$.

Na Figura 12 comparamos a evolução da produção, quando injetamos somente água com polímeros e quando injetamos um banco de água seguido de água com polímeros. Observamos que a produção é um pouco maior no inicio $\left(t_{D}=1\right)$, mas no final é praticamente a mesma. Matematicamente poderíamos dizer que temos uma vantagem com relação a custo de produção, já que gastamos menos polímeros para recuperar a mesma quantidade de óleo em um mesmo espaço de tempo.

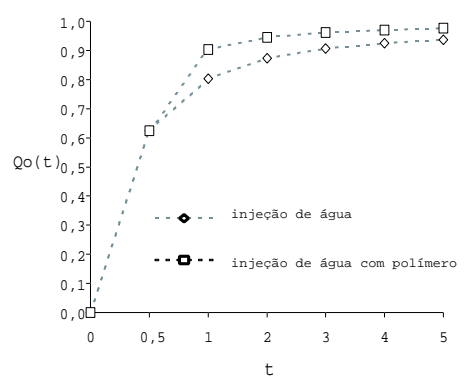

Figura 11

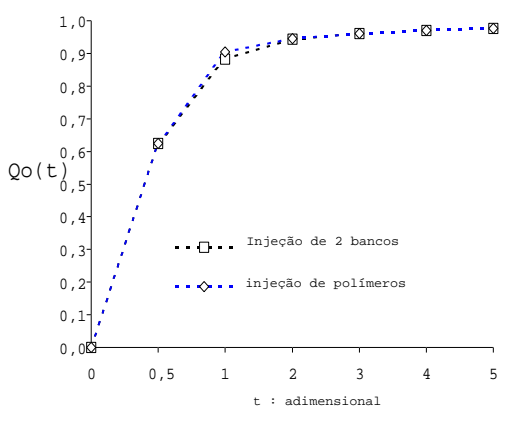

Figura 12

\subsection{Experimento 3: Influência do tamanho do primeiro banco}

No caso da injeção de bancos, o valor de $t_{0}$ (tamanho do primeiro banco) desempenha papel importante para uma análise da produção de óleo. Vamos destacar este fato neste experimento, analisando a produção de óleo depois que um volume poroso de fluido foi injetado $\left(t_{D}=1\right)$.

Fazendo variar o valor de $t_{o}$, verificamos que a quantidade de óleo produzida é para $t_{o}=0.1$ é maior que a quantidade produzida para $t_{o}=0.8$. O fato é que quando $t_{o}=0.1$ estamos injetando pouca água e bastante polímero e a produção é dominada pela injeção de polímero. Para $t_{o}=0.8$, o processo está sendo dominado pela injeção de água que recupera uma quantidade menor. A Figura 13 mostra o gráfico da produção de óleo $Q_{o}(1)$ (um volume poroso injetado) para diversos valores de $t_{o}$. 
Observamos através do gráfico, que durante o processo de recuperação, se já tivermos injetado $50 \%$ do volume poroso de água no reservatório não seria interessante injetarmos a mistura de água com polímero, pois isso não acarretará em um aumento da produção de óleo quando $t_{D}=1$.

\subsection{Experimento 4: Comparação com o método numérico}

O propósito deste experimento é comparar a solução obtida pelo método semi analítico descrito neste trabalho com a solução calculada por um esquema de diferença finitas de primeira ordem tipo upwind como sugerido por Tveito em [7].

Vamos considerar o caso em que tres bancos são injetados. Neste caso a solução apresenta quatro descontinuidades na solução mostradas na Figura 14. Podemos observar que a solução numérica suaviza as descontinuidades deixando de mostrar certas particularidades da solução.

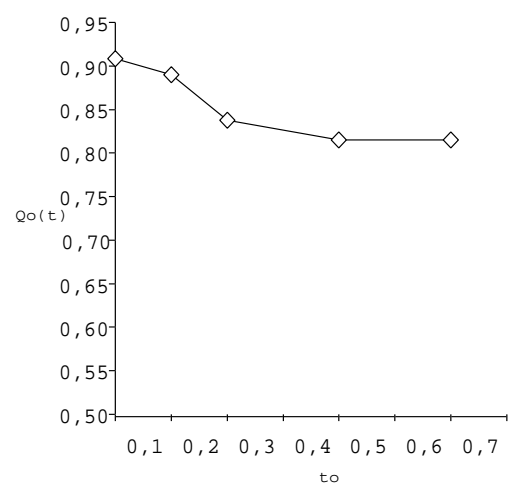

Figura 13

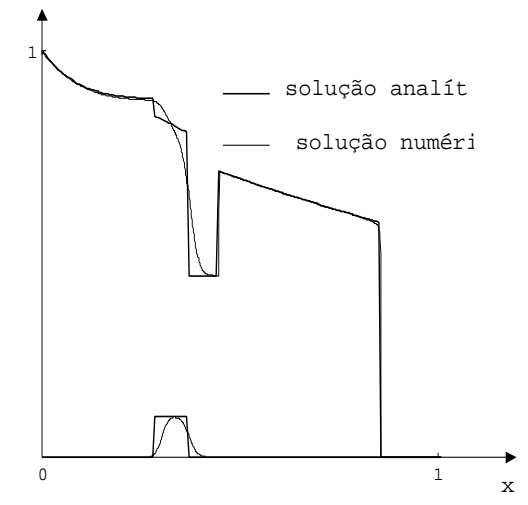

Figura 14

Na Figura 15 apresentamos os caminhos da solução obtida no espaço de estados. Podemos notar que as soluções numéricas em $s \times c$, converge para a solução semianalítica apresentada. De acordo com Le Veque [5] e Crandall [1] podemos afirmar que a solução semi-analítica obtida é a solução fisicamente (ou solução de entropia) correta uma vez que o método numérico utilizado é um método de primeira ordem monótono e convergente.

Assumimos que os parâmetros da malha satisfazem à condição de estabilidade CFL (Condição de Courant-Friedrichs-Lewy) dada em [5]. Para a função de fluxo fracionário que estamos utilizando podemos considerar o valor $C F L=12 / 25$ conforme Johansen [3]. 


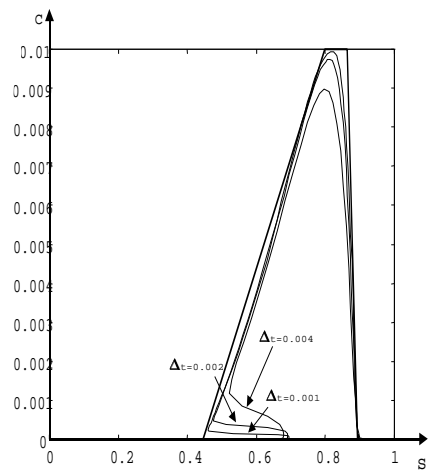

Figura 15

\section{Conclusões}

O propósito deste trabalho foi a implementação e elaboração de algoritmos para a solução numérica do sitema de equações que modela o problema da recuperação de petróleo através da injeção de água, injeção de água com polímeros e principalmente o da injeção de bancos.

Para o problema da injeção de bancos, que é caracterizado por um sistema de equações diferenciais parciais com condições iniciais e de contorno dadas, desenvolvemos um método, onde utilizamos as soluções do problema de Riemann para a localização das curvas de descontinuidades que aparecem no problema. Esta também é a idéia dos métodos Front-Tracking. A diferença é que nas regiões de suavidade utilizamos também o método das características.

Para a elaboração a apresentação dos algoritmos utilizamos as soluções apresentadas por Isaacson [2], Os algoritmos localizam as descontinuidades da solução no plano $x \times t$, através do estudo da interação entre ondas de choque e de rarefação que aparecem na solução do problema de Riemann.

Comparamos o método semi-analítico estudado com um método numérico de primeira ordem ([7]) e realizamos alguns experimentos comparando os processos de recuperação citados. Nesta comparação analisamos o comportamento da solução no plano das fases $s \times c$ e vimos através de exemplos que a solução obtida pelo método numérico converge para a solução semi-analítica.

A implementação da solução utilizando o método semi-analítico é elaborada, pois envolve o estudo da solução do Problema de Riemann associado.

Os programas computacionais foram elaborados dentro do ambiente MATLAB, o qual possui uma linguagem própria próxima da linguagem C. Várias rotinas do MATLAB facilitaram a implementação do nosso algoritmo. Destaque também para a parte gráfica que nos permitiu uma melhor visualização das soluções calculadas. 


\section{Nomenclatura}

Apresentamos aqui algumas nomenclaturas importantes do texto.

$s_{o}$ : saturação inicial adimensional no reservatório.

$s_{1}$ : saturação de água durante a injeção do primeiro banco.

$s_{2}$ : saturação de água durante a injeção do segundo banco.

$s_{w}$ : saturação de água dimensional.

$s_{w i}$ : saturação de água inicial.

$s_{o r}$ : saturação de óleo residual.

$c_{o}$ : concentração de polímero no interior do reservatório.

$c_{1}$ : concentração de polímero durante a injeção do primeiro banco.

$c_{2}$ : concentração de polímero durante a injeção do segundo banco.

$f(s, c)$ : função de fluxo fracionário.

$u_{d}$ : estado à direita no problema de Riemann.

$u_{e}$ : estado à esquerda no problema de Riemann.

\section{Referências}

[1] M.G. Crandall, A. Majda, Monotone difference approximations for scalar conservation laws. Mathematics of Computation, 24 No.149 (1980), 1-21.

[2] E.L. Isaacson, Global solution of a Rieman Problem for a non strictly hyperbolic system of conservation laws arising in enhanced oil recovery. Rockefeller University preprints, 1980.

[3] T. Johansen, R. Winther, The solution of Riemann Problem for a hyperbolic system of conservation laws modeling polymer flooding. SIAM - J. Math. Anal., 19 (1988), 541-566.

[4] L.W. Lake, "Enhanced Oil Recovery", Prentice Hall, New Jersey, 1989.

[5] R.J. Le Veque, "Numerical Methods for Conservation Laws", Birkhaüser Verlag, 1990.

[6] C.M. Marle, "Multiphase Flow in Porous Media", Editions Technip, Paris, 1981.

[7] A. Tveito, R. Winther, Convergence of a non conservative finite difference scheme for a system of hyperbolic conservation laws. Dif. Integral Equations, 3 No.5 (1990), 979-1000.

[8] M. Walsh, L.W. Lake, Applying fractional flow theory to solvent flooding and chase fluids. Journal of Petroleun Science and Engineering, 2 (1989), 281-303. 
